\title{
Photoplethysmographic Waveforms Recorded from Patients with Untreated Hypertension
}

\author{
Yanchun $\mathrm{Hu}^{1}$, *, Yanxia $\mathrm{Hu}^{2}$, Shenju Song ${ }^{3}$ \\ ${ }^{1}$ Yanchun Hu, The Fifth People's Hospital, Jinan City, China \\ ${ }^{2}$ Yanxia Hu, Center for Disease Control and Prevention, Jinan City, China \\ ${ }^{3}$ Shenju Song, The Fifth People's Hospital, Jinan City, China \\ Email address: \\ lixing777521@163.com (Yanchun Hu), yutaotai71@sina.com (Yanchun Hu)
}

\section{To cite this article:}

Yanchun Hu, Yanxia Hu, Shenju Song. Photoplethysmographic Waveforms Recorded from Patients with Untreated Hypertension. Clinical Medicine Research. Vol. 4, No. 5, 2015, pp. 163-167. doi: 10.11648/j.cmr.20150405.18

\begin{abstract}
The goal of this study was to establish whether changes in microvascular perfusion play an important role in the development of hypertension. To achieve this goal, we measured the photoplethysmographic waveforms recorded from the fingertips of patients with untreated hypertension. The photoplethysmographic waveforms were obtained from 60 healthy, normotensive male controls (NTs) and 30 untreated hypertensive male patients (HTs). The NTs were divided into two groups: a younger NT group (age 20 to 40 years, 30 individuals) and an older NT group (age 40 to 60 years, 30 individuals). The PPG waveform displayed a steep rise and a notch on the falling slope in the younger NT group. And for younger subjects, there were two positive waves (P1 and P2) and one negative wave (V).In the older NT group, a more gradual rise and fall was observed. For the HT group, no pronounced dicrotic notch was observed. A program was used to calculate the $\mathrm{P} 2 / \mathrm{P} 1$ and $\mathrm{P} 2 / \mathrm{V}$ ratios. The $\mathrm{P} 2 / \mathrm{P} 1$ and $\mathrm{P} 2 / \mathrm{V}$ ratios can indicate the perfusion to the fingertips during cardiac diastole. There were no significant differences in $\mathrm{P} 2 / \mathrm{P} 1$ between the older NT group and HTs $(0.37 \pm 0.07$ versus $0.33 \pm 0.05, \mathrm{p}>0.05)$. The $\mathrm{P} 2 / \mathrm{V}$ ratios were significantly different between the older NT group and HTs $(0.93 \pm 0.11$ versus $0.59 \pm 0.08, \mathrm{p}<0.05)$. These results indicate that there is a decrease in perfusion to the fingertips in hypertensive subjects during cardiac diastole.
\end{abstract}

Keywords: Hypertension, Microcirculation, Systemic Vascular Resistance, Endothelium, Arteriovenous Anastomoses, Photoplethysmography

\section{Introduction}

Microcirculation is the circulation of the blood in the smallest blood vessels, composed of terminal arterioles, capillaries and venules which drain capillary blood. Capillaries, which are about 5-8 $\mu \mathrm{m}$ in diameter, have no smooth muscle. Red blood cells with diameters of $7 \mu \mathrm{m}$ must deform to pass through them. Arterioles, which are 10-100 $\mu \mathrm{m}$ in diameter, are surrounded by smooth muscle cells. From a clinical viewpoint, the relaxation of vascular smooth muscles could decrease systemic vascular resistance and have an antihypertensive effect. Exactly,of all of the factors that effect resistance, the radius of the vessel is the most potent. Poiseuille's law states that resistance varies inversely as the fourth power of the radius of the vessel. This means that if the radius of a vessel doubles, then the resistance will be reduced 16 fold. Because flow resistance is caused by friction between moving fluid and vessel walls, friction between the blood and capillary wall should not be overlooked [1].To establish a relationship between the resistance of capillaries and blood pressure, it is necessary to study microvascular perfusion in detail. Photoplethysmography (PPG) is a simple, low-cost optical technique that can be used to detect changes in blood volume in the microvascular tissue bed. Analysis of PPG waveforms can help us to obtain information regarding microcirculation [2]. Valuable previous research has been conducted to detect the peaks and valleys of PPG signals. To establish whether changes in microvascular perfusion play an important role in the development of hypertension, we studied photoplethysmographic waveforms recorded from the fingertips of patients with untreated hypertension and compared the shapes of the waveforms with those obtained for different study groups. 


\section{Materials and Methods}

\subsection{Measurement System}

For photoplethysmographic (PPG) measurements, a finger clip sensor (PHILIPS Sure Signs VM8 Multi-Parameter Monitor) was used. A pulse oximeter allows the passage of infrared light into peripheral organ and measures changes in light absorption during the pulsatile cycle. The finger PPG was measured in the transmittance mode using an $810-\mathrm{nm}$ near-infrared light-emitting diodes (LED) as the light source. The bandwidth of filtering in PPG system was $0.5-20 \mathrm{~Hz}$. All signals were sampled at a rate of $1 \mathrm{kHz}$ with a resolution of 16 bits using an analog-to-digital converter. During systole, a new pulse of arterial blood enters the vascular bed, and both the blood volume and the light absorption increase. During diastole, the blood volume and light absorption reach their lowest point [3].

\subsection{Subjects}

This study was conducted in 60 healthy, normotensive male controls (NTs) and 30 untreated hypertensive male patients (HTs). The NTs were divided into two groups: a younger NT group (age 20 to 40 years, 30 individuals) and an older NT group (age 40 to 60 years, 30 individuals). The inclusion criteria for all of the participants were an age of 20 to 60 years, BMI of 19 to $24.9 \mathrm{~kg} / \mathrm{m}^{2}$, heart rate of 60 to $85 \mathrm{BPM}$, no diabetes, no illicit drug use, and an ability to understand the study protocol. Hypertensive patients could be included if they had stage 1 to 2 hypertension (blood pressure $\geq 140 / 90$ and $<180 / 110 \mathrm{mmHg}$, age 40 to 60 years). The baseline blood pressure of HT patients was the average daytime blood pressure recorded during a 24-hour period of ambulatory blood pressure measurements. For the NTs, normal blood pressure was defined as an average of 6 office measurement $\leq 135 / 88 \mathrm{mmHg}$ on 2 different occasions. All of the participants should refrain from taking all medications for $\geq 4$ times half-lives of the drug prior to the PPG measurements.

The exclusion criteria for all participants were the presence of arrhythmia, diabetes, renal disease, Raynaud's disease, and a pacemaker or other metallic implanted device. After explaining the nature and purpose of the study, informed written consent was obtained from each subject. The protocol was approved by the local research review committee (Medical ethics committee, Jinan).

Measurements were performed in a laboratory (room temperature, 20 to 25 degrees Centigrade). Before the measurements were performed, each subject was asked to relax, sit on a chair and rest his forearm on the laboratory table to help maintain a steady hand for 10 minutes. An operator then attached the finger sensor to the fourth finger tip of the left hand. It is important to have a comfortable arm position to keep the finger relatively motionless to obtain a stable and repeatable recording. During the measurements, the subjects breathed gently and did not gasp or yawn. The length of the recorded signal was 5 minutes.

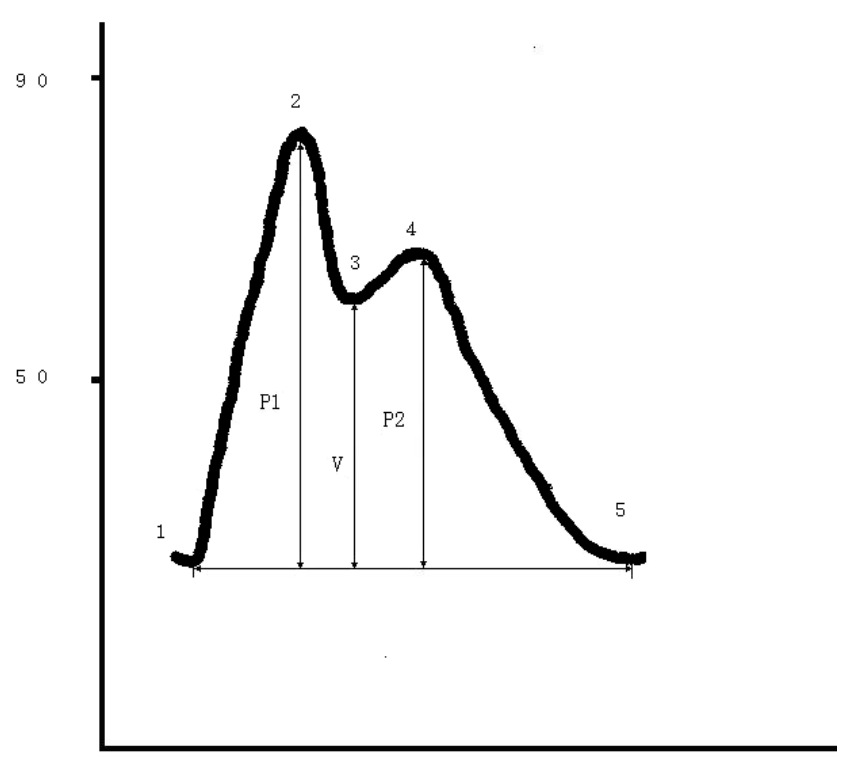

Figure 1. Schematic diagram of $P P G$ waveform.

\subsection{Physiological Measurements and Analysis}

The PPG waveform comprises a pulsatile ('AC') physiological waveform that is attributed to cardiac synchronous changes in blood volume with each heartbeat. When more blood is present in the microvascular bed, more light is absorbed [4]. For younger subjects, there were two positive waves (P1 and P2) and one negative wave (V), as shown in figure 1. (Figure 1 Example of PPG waveform recognition) The minimum and maximum threshold (P1 and $\mathrm{P} 2)$ and valley (V) values were used as markers for the signal analysis and comparison. To locate the necessary points of the signal, five markers were used. Marker 1 was the first foot of the PPG waveform; Marker 2 was the first peak of the waveform; Marker 3 was the valley; Marker 4 was the second peak; and Marker 5 was the second foot of the waveform [5].

The analysis program enabled us to calculate the peak ratio (P2/P1) (amplitude foot-to-peak 1, giving P1; amplitude foot-to-peak 2, giving $\mathrm{P} 2$ ) and the $\mathrm{P} 2 / \mathrm{V}$ ratio (amplitude foot-to-peak 2, giving P2; amplitude foot-to-valley, giving V). Sixty-second epochs of the PPG signals were manually extracted for the analysis. Using each foot as a reference, the foot-to-peak amplitudes of the first and second peaks were calculated beat-by-beat. For each subject, the mean of each complete pulse was calculated and included in the analysis. Next, the P2/P1 and P2/V ratios were calculated. Data were analyzed using Microsoft Excel and the SPPS 17.0 software package for Windows. Data are expressed as the mean \pm SD. Comparisons between means of continuous variable were performed using Student's $t$ test as appropriate. A $p$ value $<0.05$ was considered statistically significant.

\section{Results}

Ninety males were included in the study. Table 1 shows the baseline characteristics of the samples used for the longitudinal analyses. The majority of the participants were 
college educated and relatively affluent. All of the participants were Asian. The groups were generally comparable across the background variables.

Table 1. Background characteristics of the Sample.

\begin{tabular}{llll}
\hline \multirow{2}{*}{ Characteristics } & Younger NT & Older NT & HTs \\
\cline { 2 - 4 } & $\mathbf{( n = 3 0 )}$ & $\mathbf{( n = 3 0 )}$ & $\mathbf{( n = 3 0 )}$ \\
\hline Sex, male & $27.9 \pm 5.5$ & $50.7 \pm 5.6$ & $51.0 \pm 5.5$ \\
Age,year & $1.73 \pm 0.05$ & $1.71 \pm 0.04$ & $1.72 \pm 0.04$ \\
Height, $\mathrm{m}^{2}$ & $65.6 \pm 6.9$ & $65.7 \pm 6.6$ & $67.7 \pm 5.0$ \\
Weigh, kg & $21.8 \pm 1.9$ & $22.2 \pm 1.8$ & $22.9 \pm 1.2$ \\
BMI, kg/ m & $71.0 \pm 4.8$ & $72.7 \pm 4.6$ & $73.8 \pm 4.9$ \\
Heart rate, BPM & $111.3 \pm 7.6$ & $116.9 \pm 8.4$ & $163.3 \pm 9.8$ \\
Systolic BP, mmHg & & & \\
Diastolic BP, mmHg & $71.6 \pm 4.8$ & $78.3 \pm 8.4$ & $98.7 \pm 4.5$ \\
MAP & & & \\
mmHg & $84.5 \pm 5.2$ & $91.1 \pm 7.9$ & $119.8 \pm 4.9$ \\
\hline
\end{tabular}

For the younger HT, each PPG waveform involved four major phases.

+ a elevation: This phase occurs between Marker 1 and Marker 2, and it correlates with the QRS segment on a synchronous ECG. This phase is primarily concerned with the left ventricular ejection. The left ventricular ejection generates arterial pressure, increasing the drop in pressure between the left ventricle and the capillaries and thus ensuring peripheral organ perfusion. Therefore, in this phase, the blood volume in the capillaries of the organs is increasing. In the younger HT, this phase (T1) lasted $0.17 \pm 0.02$ seconds.

- b descent: This phase occurs between Marker 2 and Marker 3. The hydrostatic pressure in the capillaries increases together with the blood volume in the capillaries of the organs. When the ventricles stop contracting and begin to relax, the drop in pressure between the left ventricle and capillaries decreases, and the blood volume pumped into the capillaries is less than that volume out of them. Therefore, in this phase, the blood volume in the capillaries of the organs is decreasing. In the younger subjects, this phase (T2) lasted $0.20 \pm 0.04$ seconds.

$+\mathrm{c}$ elevation: This phase occurs between Marker 3 and Marker 4. Because the ventricles begin to relax, the drop in pressure between the arteries and capillaries decreases in the $\mathrm{b}$ descent phase. However, in the $+\mathrm{c}$ elevation, more blood is again present in the microvascular bed. The cause of this wave requires further analysis. In the younger subjects, this phase (T3) lasted $0.18 \pm 0.05$ seconds.

-d descent: This phase occurs between Marker 4 and Marker 5. Because the driving force has decreased, less blood is present in the microvascular tissue bed. In the younger subjects, this phase (T4) lasted $0.29 \pm 0.04$ seconds.

The differences in the signal shape can be easily observed visually in Figure 2. (Figure 2 Differences in signal shape. There are two peaks in the younger NT group. The HTs show a -c descent.) In the younger HT group, the signal showed a steep rise and a notch on the falling slope. In the older HT group, a more gradual rise and fall was observed. In the HTs, no pronounced dicrotic notch was observed. None of the HTs showed a true $+c$ elevation; instead, we observed $a-c$ descent in these subjects. Therefore, in hypertensive subjects, Marker
3 characterizes the location of the midpoint from Marker 1 to the next wave, and Marker 4 characterizes the location of the midpoint from Marker 2 to Marker 5.The P2/P1 ratios of 3 groups(Y NT,O NT and HTs) are $0.44 \pm 0.11,0.37 \pm 0.07$ and $0.33 \pm 0.05$.The $\mathrm{P} 2 / \mathrm{V}$ ratios of 3 groups(Y NT,O NT and HTs) are $1.29 \pm 0.15,0.93 \pm 0.11$ and $0.59 \pm 0.08$.

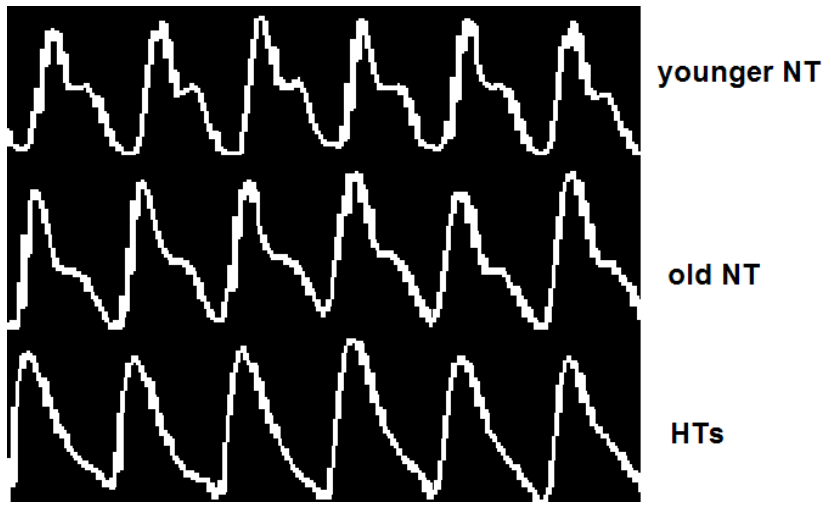

Figure 2. Differences in signal shape. There are two peaks in the younger NT group. The HTs show a-c descent.

Differences in the $\mathrm{P} 2 / \mathrm{P} 1$ and $\mathrm{P} 2 / \mathrm{V}$ ratios between the study groups (O NT and HTs) are shown in Table 2. The $P$ column shows a $P$-value (probability value) for rejection of the null hypothesis with a parameter of zero (i.e., not a significant linear factor). There were no significant differences in $\mathrm{P} 2 / \mathrm{P} 1$ between the older NT group and HTs $(0.37 \pm 0.07$ versus $0.33 \pm 0.05, p>0.05)$. The $\mathrm{P} 2 / \mathrm{V}$ ratios were significantly different between the older NT group and HTs $(0.93 \pm 0.11$ versus $0.59 \pm 0.08, p<0.05)$. These results indicate that the $\mathrm{P} 2 / \mathrm{V}$ ratio can be used to analyze blood volume changes in the microvascular tissue bed.

Table 2. Differences in the P2/P1and P2/V ratios between groups.

\begin{tabular}{llll}
\hline & Older NT & HTs & $P$ value \\
\hline $\mathrm{P} 2 / \mathrm{P} 1$ & $0.37 \pm 0.07$ & $0.33 \pm 0.05$ & $>0.05$ \\
$\mathrm{P} 2 / \mathrm{V}$ & $0.93 \pm 0.11$ & $0.59 \pm 0.08$ & $<0.05$ \\
\hline
\end{tabular}

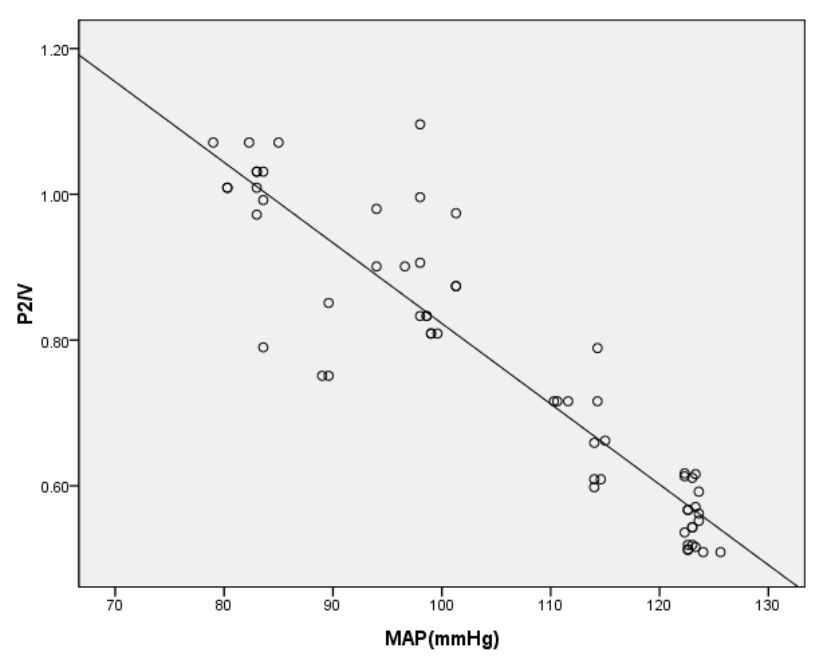

Figure 3. Scatterplots showing the relationship between the mean arterial pressure and P2/V in the old NT group and HTs. 
The mean arterial pressure (MAP) is equal to the diastolic pressure plus $1 / 3$ of the pulse amplitude.Figure 3 shows the relationship between the mean arterial pressure and $\mathrm{P} 2 / \mathrm{V}$ in 2 groups (O NT group and HTs). (Figure 3 Scatterplots showing the relationship between the mean arterial pressure and $\mathrm{P} 2 / \mathrm{V}$.)

\section{Discussion}

\subsection{The Dicrotic Notch}

It is generally accepted that PPG signal components can provide valuable information about the cardiovascular system; however, the origins of these components are not fully understood [2]. The PPG waveform was used to detect changes in blood volume in the microvascular tissue bed. If the blood volume in the microvascular tissue bed increases, then an elevated PPG waveform is observed [6]. In younger NTs, the incident wave ( + a elevation) is concerned with the left ventricular ejection.Because of the ventricular contraction, which increases arterial blood pressure, a volume of blood is squeezed into the periphery, and the blood volume pumped into is greater than that pumped out of the microvascular beds. Thus, + a elevation is involved in the PPG waveform, which indicates that the microvascular bed allows the partial storage of blood injected from arteries due to the elastic response of the vessel walls [7].

When the ventricles stop contracting and begin to relax, the arterial blood pressure decreases, and the blood volume pumped into is less than that pumped out of the microvascular beds. As a result, there is a sharp - b descent in the PPG waveform.

In the younger NTs, there were two peaks in the PPG waveform. The second peak of the PPG represents an increase in blood volume in the microvascular bed during diastole. As shown in the PPG waveform, when the ventricles begin to relax, the arterial blood pressure decreases and organ perfusion is reduced [8]. The following question remains: how does one interpret the product of the second peak of the PPG waveform?

It is easy to associate the second peak of the PPG waveform with the second peak of the arterial pulse waveform[9]. In younger subjects, the features of the PPG waveform were very similar to those of the pulse waveform. However, it differed from the pulse waveform in terms of the type of volume fluctuation. If the second peak of the PPG waveform was due to the arterial pulse waveform, then doubt remains. Because the elastic modulus of the large artery is constant, - b descent (T3) will be relatively constant. If the heart rate of younger subjects increase (such as after running), the cardiac cycle will shorten. In addition, the second peak and next heartbeat will overlap, and the waveform should become irregular. However, in fact, irrespective of the change in the heart rate of younger subjects, the PPG waveform remains uniform [10].

\subsection{Hypertension}

The results of this study revealed a significant difference in the position of the second peak between NTs and HTs. The PPG waveform of the HTs lacked the second peak. Most investigators have stated that this result is due to an increase in arterial stiffness. Arterial stiffness increases with age and results in an increase in blood pressure [11]. However, in the present study, some of the younger subjects with high blood pressure without an increase in arterial stiffness (measured by the pulse wave velocity) did not display the dicrotic notch on the PPG waveform. In addition, controlled hypotension has been used to reduce bleeding during surgery. For most hypertensive patients during surgery, the PPG waveforms are more similar to those observed for healthy older subjects; during controlled hypotension, some patients even have a second peak similar to that observed in younger subjects [12].

Cardiac output is one determinant of arterial pressure. Due to the elastic properties of the arterial walls, not all cardiac output flows through the microvascular bed during systole; a large portion of the blood flows through the microvascular bed in diastole [13]. For hypertensive patients, microvascular perfusion during diastole is significantly different from that of normal people. Antihypertensive drugs can significantly improve microvascular perfusion. Patients on antihypertensive drugs have PPG waveforms that appear to be more similar to those of healthy older subjects, and their blood pressure gradually decreases [14].

\subsection{Arteriovenous Anastomoses}

Left ventricular ejection can generate an intravascular pressure difference and increase blood flow velocity. During ventricular diastole, another potential route to generate a difference in intravascular pressure is via a decrease in peripheral resistance. According to current knowledge, arteriovenous anastomoses (AVAs) are low-resistance vessels. They have a considerably larger diameter than capillaries, and they constitute low-resistance channels by which the high-capacity venous bed can be rapidly perfused [15]. In contrast to capillaries, which provide nutrition to tissues, arteriovenous anastomoses are believed to play a thermoregulatory role in most regions of the skin. In humans, sweating is the primary means of thermoregulation. The evaporation of sweat has a cooling effect. However, the sweat glands cannot secrete this fluid without microvascular perfusion. In hot conditions, during which arteriovenous anastomoses are open, dramatic elevations in blood flow in the skin can occur [16]. However, if arteriovenous anastomoses open during systole, capillary perfusion decreases, which influences the secretion of sweat. [17].

Arteriovenous anastomoses have been studied previously using radioactive microspheres and electromagnetic techniques; however, it is likely that they have been overlooked. Histologically, AVAs are characterized by an irregular lumina (3-8 times larger than those of capillaries) and muscular walls. In cross-sections, the thick walls of the arteriovenous anastomoses consist of circular and longitudinally arranged smooth muscle fibers [18]. The opening is usually narrow and has a stellate outline due to the ridges of smooth muscle cells that protrude into the lumen. There is a notable thickening of the vascular smooth muscle at the origin of AVAs from the parent arterioles. 
The thickening tissue of the vascular smooth muscle in the parent arterioles makes it possible to forms a lip-type structure. For the parent artery, the vascular smooth muscle differs from the endothelial cells in the elastic modulus [19]. The vascular wall tension of the parent artery varies with the intravascular pressure over the cardiac cycle. Therefore, it is structurally possible for the AVAs to be closed during systole (blood pressure $\geq 90 \mathrm{mmHg}$ ) and to be open during diastole (blood pressure $<90 \mathrm{mmHg}$ ) due to higher and lower wall tension in the arterioles, respectively. In fact, if the systolic blood pressure becomes reduced $(<90 \mathrm{mmHg})$ due to bleeding during shock, then the arteriovenous anastomoses will open. This phenomenon is characteristic of changes in the microcirculation during shock [20].

Vascular endothelial dysfunction represents an initial step toward hypertension. The endothelium plays multiple pathological and physiological roles, including the regulation of smooth muscle tone. Vascular smooth muscle relaxation or contraction may result in wall tension in arterioles, which also leads to arteriovenous anastomoses [21].

\subsection{Summary}

The findings of the present study provide important information for the further investigation of microcirculation. In ventricular diastole, blood perfusion of the fingertip in hypertensive patients is significantly different from that of normal people. Changes in microvascular perfusion during diastole are not only characteristic of hypertension, but these changes likely play an important role in the development of hypertension. Photoplethysmographic (PPG) technology could be used to develop an understanding of the underlying physiology responsible for the waveform. Because the information provided by PPG waveforms is of such great importance and is often consulted in clinical decision-making, it will be to our advantage to fully appreciate all of the information provided by PPG waveforms.

\section{References}

[1] Yanchun H. Shenju S. Yanxia H. Flow Resistance of Vessels with an Enlarged Total Cross-Sectional Area in the Midsection. The Open Circulation \& Vascular Journal. 2013, 6 9-12.

[2] Allen J.Photoplethysmography and its application in clinical physiological measurement. Physiol Meas. 2007 Mar; 28(3):R1-39. Epub 2007 Feb 20.

[3] Shelley KH. Photoplethysmography: beyond the calculation of arterial oxygen saturation and heart rate. Anesthesia and analgesia. 2007 Dec; 105(6 Suppl):S31-6, tables of contents.

[4] K Abo Alam. Fuzzy Logic Hemoglobin Sensors. Online-Ressource.2011.

[5] Middleton PM, Chan GS, Steel E.Fingertip photoplethysmographic waveform variability and systemic vascular resistance in intensive care unit patients. Med Biol Eng Comput. 2011 Aug; 49(8):859-66. Epub 2011 Feb 22.

[6] Allen J, Murray A. Age-related changes in the characteristics of the photoplethysmographic pulse shape at various body sites.
Physiol Meas. 2003 May; 24 (2):297-307.

[7] Kielty CM, Stephan S, Sherratt MJ, Williamson M, Shuttleworth CA. Applying elastic fibre biology in vascular tissue engineering. Philos Trans R Soc Lond B Biol Sci. 2007 Aug 29; 362(1484):1293-312.

[8] Aymen A Awad, Ala S Haddadin. The relationship between the photoplethysmographic waveform and systemic vascular resistance. Journal of Clinical Monitoring and Computing. 01/2008; 21(6):365-72. DOI: 10.1007/s10877-007-9097-5.

[9] Agnoletti D, Millasseau SC, topouchian J. Pulse wave analysis with two tonometric devices: a comparison study. Physiol Meas. 2014 Sep; 35(9):1837-48.doi: 10.1088/0967-3334/35/9/1837. Epub 2014 Aug 26.

[10] Lee QY, Chan GS, Redmond SJ, Middleton PM. Multivariate classification of systemic vascular resistance using photoplethysmography. Physiol Meas. 2011 Aug; 32(8):1117-32. doi: 10.1088/0967-3334/32/8/008. Epub 2011 Jun 21.

[11] O'Rourke MF, Hashimoto J. Mechanical factors in arterial aging: a clinical perspective. J Am Coll Cardiol. 2007 Jul 3; 50(1):1-13.

[12] London GM, Pannier B. Arterial functions: how to interpret the complex physiology. Nephrol Dial Transplant. 2010 Dec; 25(12):3815-23.

[13] Blacher J, Protogerou AD, Safar ME. Large artery stiffness and antihypertensive agents. Curr Pharm Des. 2005; 11(25):3317-26. Review.

[14] Middleton PM1, Chan GS, Steel E. Fingertip photoplethysmographic waveform variability and systemic vascular resistance in intensive care unit patients. Med Biol Eng Comput. 2011 Aug; 49(8):859-66. doi: 10.1007/s11517-011-0749-8. Epub 2011 Feb 22.

[15] Rozen WM, Chubb D. Macrovascular arteriovenous shunts (MAS): a newly identified structure in the abdominal wall with implications for thermoregulation and free tissue transfer. $\mathrm{J}$ Plast Reconstr Aesthet Surg. 2010 Aug; 63(8):1294-9. Epub 2009 Jul 3.

[16] Hales JR, Jessen C, Fawcett AA, King RB.Skin AVA and capillary dilatation and constriction induced by local skin heating. Pflugers Arch. 1985 Jul;404(3):203-7.

[17] Hales JR, Fawcett AA, Bennett JW, Needham AD. Thermal control of blood flow through capillaries and arteriovenous anastomoses in skin of sheep. Pflugers Arch. 1978 Dec 15;378(1):55-63.

[18] Midtgård U. Development of arteriovenous anastomoses in the skin of the chicken and the influence of environmental temperature. Am J Anat. 1989 Nov; 186 (3):300-5.

[19] Yanchun H. Shenju S. Lijun Z. Jilie C. Hypertension and microcirculation shunt vessel. China Foreign Medical Treatment.2009.19.002

[20] Kochetygov NI, Pozdniakov PK. Hemodynamics, arteriovenous anastomoses and oxygen regimen during severe blood loss and infusion therapy. Patol Fiziol Eksp Ter. 1981 Jul-Aug; (4):32-7.

[21] Shafique M, Kyriacou PA, Pal SK. Investigation of photoplethysmographic signals and blood oxygen saturation values on healthy volunteers during cuff-induced hypoperfusion using a multimode $\mathrm{PPG} / \mathrm{SpO}(2)$ sensor.Med Biol Eng Comput. 2012 Jun; 50(6):575-83. Epub 2012 May 4. 